Max-Planck-Institut für demografische Forschung

Max Planck Institute for Demographic Research

Konrad-Zuse-Strasse 1 - D-18057 Rostock - GERMANY

Tel +49 (0) 3812081 - 0; Fax +49 (0) 3812081 - 202;

http://www.demogr.mpg.de

MPIDR TECHNICAL REPORT 2015-004

JUNE 2015

\title{
Reading Human Fertility Database and Human Mortality Database data into $\mathbf{R}$
}

Tim Riffe

For additional material see www.demogr.mpg.de/tr/

This technical report has been approved for release by: Vladimir Shkolnikov (shkolnikov@demogr.mpg.de), Head of the Laboratory of Demographic Data.

(C) Copyright is held by the authors.

Technical reports of the Max Planck Institute for Demographic Research receive only limited review. Views or opinions expressed in technical reports are attributable to the authors and do not necessarily reflect those of the Institute. 


\title{
Reading Human Fertility Database and Human Mortality Database data into $R$
}

\author{
Tim Riffe \\ Department of Demography, University of California, Berkeley
}

June 1, 2015

\begin{abstract}
The features and usage of the HMDHFDplus package are demonstrated for reading data from the Human Mortality Database, the Human Fertility Database, and other similarly formatted sources directly from the database Websites into R.
\end{abstract}

\section{Motivation}

The Human Fertility Database (2015) and Human Mortality Database (2015) are two widely used data sources for the comparative and historical study of fertility and mortality. Both databases offer the option to download data in bulk in a few different formats. After a bulk download, users can then set up local databases in a variety of convenient ways, such as that described by Minton (2015) for R (R Development Core Team 2012) users. Another option for R users is to read data directly into an interactive session from the respective database websites. This is handy for small examples, lightweight reproducibility, and rapid prototyping. The HMDHFDplus package provides easy direct access to the databases using a simple standard set of arguments. Issues such as authentication and fixing column classes are handled automatically. Analogous functions are also made available for selected databases using similar formatting standards. At this time, these databases include the Japanese Mortality Database (2015), the Canadian Human Mortality Database (2015), and the Human Fertility Collection (2015). ${ }^{1}$ This report outlines the basic features and provides usage examples.

\footnotetext{
${ }^{1}$ The Human Life-Table Database (2015) may also be incorporated in the future.
} 


\section{Installation}

The HMDHFDplus package is hosted on and can be installed directly from github. ${ }^{2}$ In Linux and similar systems, first open the Terminal and run:

sudo apt-get install libcurl

sudo apt-get install libxml2-dev

Then install R's XML and RCurl packages in the usual way. Windows users get the above installations automatically with a proper installation of the devtools package. ${ }^{3}$ Users from all operating systems then install the HMDHFDplus package for $\mathrm{R}$ using:

library (devtools)

install_github ("timriffe/TR1/TR1/HMDHFDplus")

Consult the README ${ }^{4}$ file on the github repository for more details and other installation methods.

\section{$3 \quad$ Usage and examples}

Load the package using:

library (HMDHFDplus)

The two main functions of interest are readHMDweb() and readHFDweb(), and both functions have the same essential arguments. These two functions only require the user to supply country codes, data item names, and database authentication parameters. ${ }^{5}$ It helps to be familiar with HMD and HFD file naming conventions. To retrieve the population codes used in any of these databases, run:

getHFDcountries()

getHFCcountries()

getHMDcountries()

get JMDprefectures()

getCHMDprovinces()

\footnotetext{
${ }^{2} \mathrm{~A}$ package snapshot is included with this article, but I encourage users to use the current build on github, as it may contain updates and bug fixes. The package is under the following url: http://github.com/timriffe/TR1/tree/master/TR1/HMDHFDplus. This report, as well as a useful README document, can be found under http://github.com/timriffe/TR1.

${ }^{3}$ Not all versions of Windows have been tested, and Mac remains untested at this writing.

${ }^{4}$ The README is at the foot of the main repository page: https://github.com/timriffe/TR1

${ }^{5}$ The HFD also allows users to extract data from former updates, which may be useful for strict replication purposes. In order to make use of this feature, users must note the 8digit date code associated with the specific country series update. By default, readHFDweb() extracts the most recent update.
} 
This returns vectors of the standard numerical or letter codes used to identify population units.

The functions used for reading data into $\mathrm{R}$ from the Web use a common set of required arguments. In interactive $\mathrm{R}$ sessions, the following will prompt the user to enter a username and password into the console (no quotes) each time the function is run:

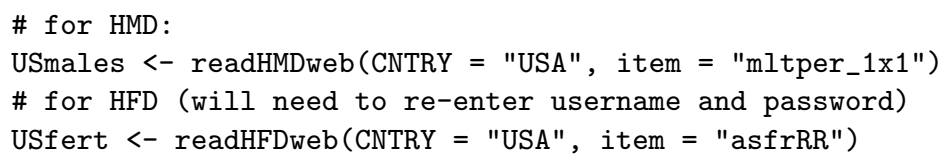

Manually entering a username and password can become tedious for larger datagrabs, so these can also be given explicitly in the arguments, like so:

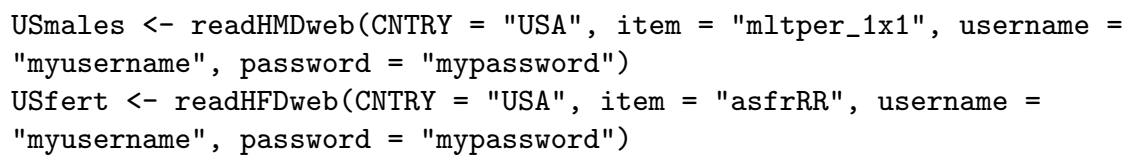

There is a security trade-off in this case, because the username and password may inadvertently be saved within your $R$ script. I suggest two alternatives in this case. First, in an interactive $\mathrm{R}$ session, define your username once at the beginning of the script, but without saving them as text within the script, like so:

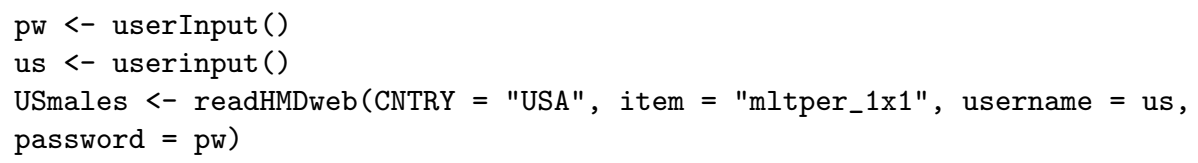

The two objects pw and us can in this case be recycled throughout the following $\mathrm{R}$ session. Second, For more frequent users, I recommend defining your HMD and HFD passwords in the .Rprofile file, such that they are defined and ready to use at the start of $\mathrm{R}$ sessions, but are not saved in your potentially-shared code. The above HMD code will return data such as the following: data.frame:

\begin{tabular}{|c|c|c|c|c|c|c|c|c|c|c|}
\hline Year & Age & $\mathrm{mx}$ & $q x$ & $a x$ & $1 \mathrm{x}$ & $d x$ & $\mathrm{Lx}$ & $\mathrm{Tx}$ & ex & OpenInterval \\
\hline 11933 & 0 & 0.06859 & 0.06515 & 0.23 & 100000 & 6515 & 94978 & 5916978 & 59.17 & FALSE \\
\hline 1933 & 1 & 0.01004 & 0.00999 & 0.50 & 93485 & 934 & 93018 & 5822000 & 62.28 & FALSE \\
\hline 31933 & 2 & 0.00467 & 0.00466 & 0.50 & 92551 & 431 & 92336 & 5728982 & 61.90 & FALSE \\
\hline 1933 & 3 & 0.00333 & 0.00333 & 0.50 & 92120 & 307 & 91967 & 5636646 & 61.19 & FALSE \\
\hline 1933 & 4 & 0.00254 & 0.00253 & 0.50 & 91814 & 233 & 91697 & 5544679 & 60.39 & FALSE \\
\hline 1933 & 5 & 0.00209 & 0.00209 & 0.50 & 91581 & 191 & 91485 & 5452982 & 59.54 & FALSE \\
\hline
\end{tabular}

This data.frame differs from the original HMD mltper_1x1 file in that the Age column is integer, and a new OpenInterval column has been added, which contains the value TRUE for age 110. HFD Age and Cohort columns are modified in a similar way for more intuitive and immediate use of these columns as integers. Likewise, abridged ages, such as "5-9" are coerced as integers of the lower interval bound, as 5. Finally, HMD Population files, obtained via 


USpop <- readHMDweb("USA", "Population", username = us, password = pw)
head(USpop)
$\begin{array}{rrrrrrrrrr}\text { Year Age OpenInterval } & \text { Female1 } & \text { Male1 } & \text { Total1 } & \text { Female2 } & \text { Male2 } & \text { Total2 } \\ 1 & 1933 & 0 & \text { FALSE } & 984472.3 & 1015362 & 1999834 & 937185.8 & 968955.4 & 1906141 \\ 2 & 1933 & 1 & \text { FALSE } & 1040496.0 & 1064088 & 2104584 & 970696.5 & 993352.8 & 1964049 \\ 3 & 1933 & 2 & \text { FALSE } & 1093043.8 & 1117527 & 2210571 & 1062002.5 & 1083452.4 & 2145455 \\ 4 & 1933 & 3 & \text { FALSE } & 1107994.3 & 1135047 & 2243041 & 1095555.1 & 1121220.2 & 2216775 \\ 5 & 1933 & 4 & \text { FALSE } & 1130624.4 & 1179514 & 2310138 & 1105999.3 & 1132665.9 & 2238665 \\ 6 & 1933 & 5 & \text { FALSE } & 1168930.6 & 1228225 & 2397156 & 1141944.4 & 1197735.4 & 2339680\end{array}$

, where columns ending in 1 indicate January 1st estimates and columns ending in 2 indicate December 31st estimates, and the Year and Age columns are coerced to an integer class. The JMD, CHMD, and HFC are all called in similar ways, but without authentication:

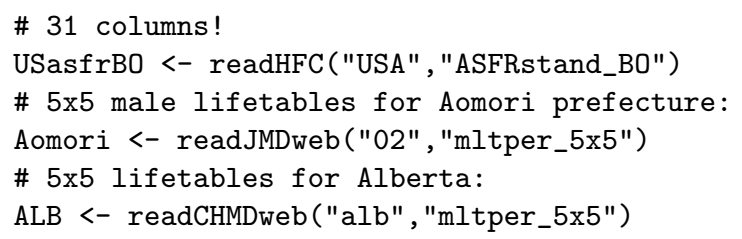

The JMD and CHMD follow the same formatting standards and naming conventions as the HMD, although the data products available are a subset of those produced by the HMD. The HFC follows different standards and conventions than the HFD.

\section{Conclusions}

Reading data directly from HMD, HFD and a selection of other databases directly from the web into $R$ is made easy with the HMDHFDplus package. At this time, utilities are provided for reading data from the HMD, JMD, CHMD, HFD, and HFC websites. Common R pitfalls are removed by coercing columns to useful classes by default.

\section{Acknowledgements}

Thanks to Joshua Goldstein and Carl Boe for supporting development of this R functionality, and to Vladimir Shkolnikov, Dmitri Jdanov, and Tomás Sobotka for the invitation to present this material at the HFD side meeting to the 2015 PAA Annual Meeting. This work was supported by the National Institute On Aging of the U.S. National Institutes of Health (NIH) under Award Numbers R01-AG011552 and R01-AG040245. The content is solely the responsibility of the author and does not necessarily represent the official views of the NIH. 


\section{References}

Canadian Human Mortality Database. Department of Demography, Université de Montréal, 2015. Available at http://www.bdlc.umontreal.ca/chmd/.

Human Fertility Collection. Max Planck Institute for Demographic Research (Germany) and Vienna Institute of Demography (Austria), 2015. Available at http://www.fertilitydata.org/cgi-bin/index.php.

Human Fertility Database. Max Planck Institute for Demographic Research (Germany) and Vienna Institute of Demography (Austria), 2015. Available at www.humanfertility.org.

Human Life-Table Database. Max Planck Institute for Demographic Research (Germany) and University of California, Berkeley (USA) and Institut national d'études démographiques (France), 2015. Available at http://www.ipss.go.jp/p-toukei/JMD/index-en.html.

Human Mortality Database. University of California, Berkeley (USA) and Max Planck Institute for Demographic Research (Germany), 2015. Available at www.mortality.org or www.humanmortality.de (data downloaded on July 10, 2014).

Japanese Mortality Database. National Institute of Population and Social Security Research (Japan), 2015. Available at http://www.ipss.go.jp/ptoukei/JMD/index-en.html.

Jon Minton. Merging, exploring, and batch processing data from the human fertility database and human mortality database. Technical Report TR-2015-001, Max Planck Institute for Demographic Research (MPIDR), April 2015. URL http://www.demogr.mpg.de/papers/technicalreports/ tr-2015-001.pdf.

R Development Core Team. R: A Language and Environment for Statistical Computing. R Foundation for Statistical Computing, Vienna, Austria, 2012. URL http://www.R-project.org/. ISBN 3-900051-07-0. 